\title{
Hitting distributions of geometric Brownian motion
}

by

\author{
T. Byczkowski and M. RYzNAR (Wrocław)
}

\begin{abstract}
Let $\tau$ be the first hitting time of the point 1 by the geometric Brownian motion $X(t)=x \exp (B(t)-2 \mu t)$ with drift $\mu \geq 0$ starting from $x>1$. Here $B(t)$ is the Brownian motion starting from 0 with $E B^{2}(t)=2 t$. We provide an integral formula for the density function of the stopped exponential functional $A(\tau)=\int_{0}^{\tau} X^{2}(t) d t$ and determine its asymptotic behaviour at infinity. Although we basically rely on methods developed in [BGS], the present paper covers the case of arbitrary drifts $\mu \geq 0$ and provides a significant unification and extension of the results of the above-mentioned paper. As a corollary we provide an integral formula and give the asymptotic behaviour at infinity of the Poisson kernel for half-spaces for Brownian motion with drift in real hyperbolic spaces of arbitrary dimension.
\end{abstract}

1. Introduction. In this paper, $B(t)$ will denote the Brownian motion starting from 0 and normalized so that $E B^{2}(t)=2 t$. Note that this normalization is different from the standard one (i.e. $E B^{2}(t)=t$ ). The reason for this choice is that after subordination (see Section 5 ) we want to obtain the appropriate version of the Poisson kernel for a half-space in $\mathbb{H}^{n}$. As a result, some formulas (e.g., in Lamperti's representation, in this section, or (5), in the Preliminaries) will have a different scaling than in the standard setup.

Now, consider the following linear SDE:

$$
d X(t)=X(t) d B(t)-(2 \mu-1) X(t) d t, \quad X(0)=x>0, \quad \mu \geq 0 .
$$

The strong unique non-exploding solution is given by

$$
X(t)=x \exp (B(t)-2 \mu t)
$$

The process $\{X(t) ; t \geq 0\}$ is called a geometric Brownian motion or exponential Brownian motion and along with the additive functional from the

2000 Mathematics Subject Classification: Primary 60J65; Secondary $60 \mathrm{J60}$.

Key words and phrases: hyperbolic spaces, stable processes, Poisson kernel.

Research partially supported by KBN grant 1 P03A 02028 and RTN Harmonic Analysis and Related Problems contract HPRN-CT-2001-00273-HARP. 
process $X(t)$,

$$
A_{x}(t)=\int_{0}^{t} X^{2}(v) d v=\int_{0}^{t} x^{2} \exp 2(B(s)-2 \mu s) d s
$$

it is of primary interest in mathematical finance and insurance theory (see, e.g., [D], [GY] or [Y2]). Also there is a connection of the above functional with the Brownian motion in hyperbolic half-spaces (see, e.g., [Y2], [AG], [BCF], [BCFY] and Section 5).

The distribution of $A_{x}(t)$ for fixed $0 \leq t \leq \infty$ has been a subject of study in a substantial number of papers (see, e.g., [B], [Y2], [AG], [M]). In this paper we investigate the properties of the density function $q_{\mu}$ of the (stopped) additive functional $A_{x}(\tau)$ where $\tau$ is the first hitting time of the point 1 by the process $X(t)$ (starting from $x>1$ ). From the strong Markov property it easily follows that the distribution of $A_{x}(\tau)$ is closely related to the distribution of $A_{x}(\infty)$. The latter is astonishingly simple; it is identical with the distribution of the random variable $x^{2} / 4 Z$, where $Z$ is a $\Gamma(\mu, 1)$-distributed random variable (with the density $\Gamma(\mu)^{-1} u^{\mu-1} e^{-u}$ ). This fundamental result is due to D. Dufresne [D] (see also [Y1], [Y2]) and is of primary importance here. Knowing the Laplace transform of $A_{x}(\infty)$ one can easily derive (via the strong Markov property) the form of the Laplace transform of the random variable $A_{x}(\tau)$ as a suitable ratio of Bessel functions. For the reader's convenience we present this argument in the Preliminaries.

On the other hand, the distributions of $A_{x}(\tau)$ and $A_{x}(\infty)$ are closely related to hitting times of Bessel processes. The main fact here is Lamperti's representation which states that there exists a Bessel process $R^{(-\mu)}$ with index $-\mu$, starting from $x>1$, such that the process $X(t)$ admits the following representation (see $[\mathrm{L}]$ and Exercise (1.28), Ch. XI, in [RY]):

$$
X(t)=R^{(-\mu)}\left(2 A_{x}(t)\right), \quad t \geq 0 .
$$

We refer the reader to [RY] for an account of Bessel processes (see also [GJY]). From the Lamperti representation it follows immediately that $A_{x}(\tau)$ and $A_{x}(\infty)$ can be regarded as hitting times of 1 and 0 , respectively, of the Bessel process $R^{(-\mu)}$ starting from $x>1$. Also it is possible to relate $A_{x}(\tau)$ and $A_{x}(\infty)$ to last exit times for appropriate Bessel processes. There is a vast literature on that subject. Such hitting or last exit times were studied by Getoor [G], Getoor-Sharpe [GS], Kent [K1]-[K3] and Pitman-Yor [PY]. A useful summary of the last paper is contained in [PY1]. Let us remark that most of the results in these papers (especially in [K1]-[K3]) are devoted to what Kent calls "left-hitting times" $\tau_{a b}$ for some Bessel processes (more generally diffusions) starting at $a$ and hitting $b$, with $0 \leq a<b<\infty$. Distributions of left-hitting times are very regular; in particular they have all moments, which makes it possible to work with their moment generating 
functions. Densities of these distributions were represented by Kent as series with exponential components (Theorem 6.1 in [K2]). In contrast, we are dealing with the so-called "right-hitting times" $\tau_{b a}$, which are much less regular. In particular, we prove in Section 4 that the densities of right-hitting times have precise asymptotics of order $t^{-\mu-1}$ for $\mu>0$, so they only have moments of order less than $\mu$. Kent's representation does not cover this case.

Let us also remark that in Getoor [G], Kent [K1] and Getoor-Sharpe [GS] the Laplace transform of the distribution of $A_{x}(\tau)$ is derived as a ratio of Bessel functions (see Preliminaries). For an exhaustive discussion on that subject we refer to [GJY].

Our main focus in this paper is to provide an integral formula for the density of $A_{x}(\tau)$ (see Theorem 3.3). In the case $\mu=1 / 2$ this density is well known to be the $1 / 2$-stable subordinator. Getoor in $[\mathrm{G}]$ writes that it is possible to obtain an explicit form of the density for $\mu=3 / 2$ but he provides neither a formula nor any details on that.

At this point let us mention that various ratios of Bessel functions have been proved to be completely monotonic functions, hence they are Laplace transforms of probability distributions (see Ismail [I1], [I2], Ismail-Kelker [IK], Kent [K1]). For a survey on this theme see Pitman and Yor [PY] and also [GY]. Regarding the ratio considered in our paper, Ismail and Kelker [IK] showed by purely analytical methods that it is an infinitely divisible distribution.

The main purpose of the paper is to obtain a representation for the density function $q_{\mu}$ of the functional $A_{x}(\tau)$ along with its asymptotic properties, for arbitrary drift terms $\mu \geq 0$. Then we apply these results to derive an integral representation of the Poisson kernel for subspaces of real hyperbolic spaces, for hyperbolic Brownian motion with arbitrary drift, which extends and simplifies the results and proofs from [BGS].

The paper is organized as follows. In the Preliminaries we collect basic information needed in the following.

In Section 3 we obtain a representation of the density of the functional $A_{x}(\tau)$. For this purpose we extend to arbitrary drifts $\mu \geq 0$ the integral representation given in [BGS] for a ratio of Bessel functions.

In Section 4 we exhibit the exact asymptotics of the density of $A_{x}(\tau)$ at infinity for all drifts $\mu \geq 0$. Again, we essentially follow the idea of [BGS]. However, applying more direct probabilistic arguments, we are able to simplify our presentation considerably, as well as cover the case of arbitrary drifts $\mu \geq 0$.

In Section 5 we show how to apply the results obtained in the preceding sections to obtain a representation and asymptotic properties of the Poisson kernel on subspaces of real hyperbolic spaces of arbitrary dimension, for hy- 
perbolic Brownian motion with drift. Some of these asymptotic properties were studied in $[\mathrm{BCF}]$ for dimension 2, where it was shown that the distribution of the Poisson kernel belongs to the stable domain of attraction. Our asymptotic results may be viewed as an extension of those in $[\mathrm{BCF}]$. For a related result see also [BCFY].

2. Preliminaries. Let $0<a<x$ and let $\tau_{a}$ be the first hitting time of the point $a$ by the geometric Brownian motion with drift $\mu \geq 0$ starting at $x$ :

$$
\tau_{a}=\inf \{t>0: x \exp (B(t)-2 \mu t)=a\} .
$$

Then $\tau_{a}<\infty$ a.e. since $\inf _{t>0} B(t)=-\infty$.

Further, define

$$
A_{x}(t)=x^{2} \int_{0}^{t} \exp 2(B(s)-2 \mu s) d s .
$$

By the strong Markov property of Brownian motion we obtain

Basic relationship (for $\mu>0)$. Observe that $x^{2} \exp 2\left(B\left(\tau_{a}\right)-2 \mu \tau_{a}\right)=a^{2}$, hence

$$
\begin{aligned}
& A_{x}(\infty)=x^{2} \int_{0}^{\tau_{a}} \exp 2(B(s)-2 \mu s) d s+x^{2} \int_{\tau_{a}}^{\infty} \exp 2(B(s)-2 \mu s) d s \\
= & A_{x}\left(\tau_{a}\right)+x^{2} \exp 2\left(B\left(\tau_{a}\right)-2 \mu \tau_{a}\right) \int_{0}^{\infty} \exp 2\left(B\left(s+\tau_{a}\right)-B\left(\tau_{a}\right)-2 \mu s\right) d s \\
= & A_{x}\left(\tau_{a}\right)+A_{a}^{\prime}(\infty)
\end{aligned}
$$

where $A_{a}^{\prime}(\infty)$ is a copy of $A_{a}(\infty)$, independent of $A_{x}\left(\tau_{a}\right)$.

The relation (1) can also be rewritten as follows:

$$
A_{x}(\infty)=A_{x}\left(\tau_{a}\right)+\frac{a^{2}}{x^{2}} A_{x}^{\prime}(\infty)
$$

The precise meaning of (2) is that the distribution of $A_{x}(\infty)$ is selfdecomposable (see, e.g., [Sa]). This relation is of prime importance when computing the asymptotic behaviour of the density function of $A_{x}\left(\tau_{a}\right)$ in Section 4.

Dufresne [D] (see also Getoor [G], Kent [K1], Getoor-Sharpe [GS], where the result is given in the context of Bessel processes) showed that the density and the Laplace transform of $A_{1}(\infty)$ are

$$
\begin{aligned}
h_{\mu}(t) & =\frac{2^{-2 \mu}}{\Gamma(\mu)} \frac{e^{-1 / 4 t}}{t^{1+\mu}}, \\
E e^{-r^{2} A_{1}(\infty)} & =2 \frac{(r / 2)^{\mu}}{\Gamma(\mu)} K_{\mu}(r), \quad \mu>0 .
\end{aligned}
$$


From this, the formula (2), and the scaling property: $A_{x}(\infty)=x^{2} A_{1}(\infty)$, one immediately obtains, by elementary properties of the Laplace transform,

$$
E e^{-r^{2} A_{x}\left(\tau_{a}\right)}=\left(\frac{x}{a}\right)^{\mu} \frac{K_{\mu}(x r)}{K_{\mu}(a r)},
$$

where $K_{\mu}$ is the modified Bessel function of second type with index $\mu$. The above Laplace transform appears in many papers (see, e.g., Kent [K1], Getoor-Sharpe [GS], Ismail-Kelker [IK]); here we followed the derivation from $[\mathrm{BCF}]$.

From the continuity of both sides of (5) with respect to $\mu \geq 0$ we infer that the above formula remains valid also for $\mu=0$.

Let us remark that for $\mu=0$ the right-hand side of (5) also gives the classical formula ([S]) for the Laplace transform of Brownian motion hitting time $T_{a}$ of the circle centred at 0 with radius $a$ in $\mathbb{R}^{2}$ from the point $y \in \mathbb{R}^{2}$ such that $|y|=x>a$.

From (5) it follows directly that for $t>0$ we have

$$
A_{t x}\left(\tau_{t a}\right) \stackrel{d}{=} t^{2} A_{x}\left(\tau_{a}\right),
$$

where $\stackrel{d}{=}$ denotes equality of distribution.

Therefore, from now on we may and do assume that $a=1$ and $x>1$ is fixed. We write $\tau$ instead of $\tau_{1}$, and $A(\tau), A(\infty)$ instead of $A_{x}\left(\tau_{1}\right), A_{x}(\infty)$, respectively.

We conclude this section with the following technical lemma:

Lemma 2.1. For $s>0$ we have

$$
\int_{0}^{\infty} e^{-y^{2} / 4 t} h_{s}\left(t / \lambda^{2}\right) d t=\frac{\lambda^{2 s+2}}{\left(\lambda^{2}+y^{2}\right)^{s}} .
$$

Proof. Indeed,

$$
\begin{aligned}
\int_{0}^{\infty} e^{-y^{2} / 4 t} e^{-\lambda^{2} / 4 t} \frac{\lambda^{2 s+2} d t}{t^{1+s}} & =\frac{\lambda^{2 s+2}}{\left(\lambda^{2}+y^{2}\right)^{s}} \int_{0}^{\infty} e^{-\left(y^{2}+\lambda^{2}\right) / 4 t} \frac{d t /\left(y^{2}+\lambda^{2}\right)}{\left(t /\left(y^{2}+\lambda^{2}\right)\right)^{1+s}} \\
& =\frac{2^{2 s} \lambda^{2 s+2}}{\left(\lambda^{2}+y^{2}\right)^{s}} \int_{0}^{\infty} \Gamma(s) h_{s}(u) d u=\frac{2^{2 s} \Gamma(s) \lambda^{2 s+2}}{\left(\lambda^{2}+y^{2}\right)^{s}} .
\end{aligned}
$$

Throughout the paper we use the following convention: by $c, C$ we always denote non-negative constants which may depend on other constant parameters only. The value of $c$ or $C$ may change from line to line in a chain of estimates.

The notation $p(u) \approx q(u), u \rightarrow u_{0}$ means that $q / p \rightarrow 1$ as $u \rightarrow u_{0}$.

3. Representation of the density of $A(\tau)$. In this section we give a representation formula for the density function of the functional $A(\tau)$, for 
arbitrary $\mu \geq 0$, where $\tau$ is the hitting time introduced in the preceding section.

We begin by stating a more general version of a lemma from [BGS]. The proof is identical and is omitted.

Lemma 3.1. Let $\mu \geq 0$ and $\lambda=x-1$. Suppose that

$$
Q(z)=z-\left(\mu^{2}-1 / 4\right) \frac{\lambda}{2 x}, \quad z \in \mathbb{C} .
$$

Define $F_{\lambda}(z)$ by

$$
\lambda F_{\lambda}(z)=\frac{z e^{\lambda z} x^{\mu} K_{\mu}(x z)-x^{\mu-1 / 2} Q(z) K_{\mu}(z)}{K_{\mu}(z)} .
$$

Then

$$
F_{\lambda}(z)=O\left(z^{-1}\right), \quad z \rightarrow \infty,
$$

and there exists a function $w_{\lambda}$ such that

$$
F_{\lambda}(z)=\int_{0}^{\infty} e^{-z v} w_{\lambda}(v) d v, \quad \Re(z)>0 .
$$

Moreover,

$$
x^{\mu-1 / 2}\left(\mu^{2}-1 / 4\right) / 2 x=\int_{0}^{\infty} w_{\lambda}(v) d v,
$$

and, for $\mu>1 / 2$,

$$
2 x^{\mu-1 / 2}=\int_{0}^{\infty} \kappa w_{\lambda}(v) d v,
$$

where $\kappa=\kappa(v)=(\lambda+v)^{2}-\lambda^{2}=v(2 \lambda+v)$.

For $\mu=1 / 2$ we have $F_{\lambda}(z) \equiv 0$.

An explicit formula for the function $w_{\lambda}$ will be provided later on.

The following formula is crucial for our considerations:

Lemma 3.2. Let $\varphi:[0, \infty) \rightarrow \mathbb{R}^{+}$belong to $L^{1}(0, \infty)$ and suppose that its Laplace transform $\hat{\varphi}$ has the following property:

$$
t^{-1 / 2} \widehat{\varphi}(t) \in L^{1}(0, \infty)
$$

Then

$$
\int_{0}^{\infty} \widehat{\varphi}\left(r^{2}\right) \cos (r y) d r=\sqrt{\pi} / 2 \int_{0}^{\infty} e^{-y^{2} / 4 t} \varphi(t) \frac{d t}{\sqrt{t}} .
$$

Proof. Let $A$ be a random variable with absolutely continuous distribution with the density function $\varphi$ and let $B(t)$ be Brownian motion starting from 0 (such that $E B^{2}(t)=2 t$ ), independent of $A$. It is easy to see that the value of the Laplace transform of $A$ at the point $r^{2}$, that is, $\widehat{\varphi}\left(r^{2}\right)$, is equal 
to the Fourier (or cosine) transform of $B(A)$ at the point $r$. Observe that our assumption ensures that this Fourier transform belongs to $L^{1}(0, \infty)$. Thus, the left-hand side of (12) is the inversion formula applied for the Fourier transform and gives the density of $B(A)$. The right-hand side results from the direct computation of this density, taking into account the independence of $B$ and $A$ and the particular form of the (gaussian) density of $B(t)$.

We are ready to state our representation formula for the density function of $A(\tau)$.

THEOREM 3.3. Let $q_{\mu}$ denote the density function of $A(\tau)$. Then for $\mu \geq 0$ we have

$$
q_{\mu}(t)=\lambda \frac{e^{-\lambda^{2} / 4 t}}{\sqrt{\pi t}}\left(x^{\mu-1 / 2} / 2 t+\int_{0}^{\infty}\left(e^{-\kappa / 4 t}-1\right) w_{\lambda}(v) d v\right),
$$

where $\kappa=\kappa(v)=(\lambda+v)^{2}-\lambda^{2}=v(2 \lambda+v)$, and $\lambda=x-1$. For $\mu>1 / 2$ we have

$$
q_{\mu}(t)=\lambda \frac{e^{-\lambda^{2} / 4 t}}{\sqrt{\pi t}} \int_{0}^{\infty}\left(e^{-\kappa / 4 t}-1+\kappa / 4 t\right) w_{\lambda}(v) d v .
$$

Proof. The proof relies on an application of the formula (12) to the function

$$
\widehat{\varphi}\left(r^{2}\right)=x^{\mu} \frac{K_{\mu}(x r)}{K_{\mu}(r)} .
$$

Note that by (7) and (10) it follows that

$$
\begin{aligned}
r x^{\mu} \frac{K_{\mu}(x r)}{K_{\mu}(r)} & =e^{-\lambda r} \lambda F_{\lambda}(r)+e^{-\lambda r} x^{\mu-1 / 2} Q(r) \\
& =e^{-\lambda r} x^{\mu-1 / 2} r+e^{-\lambda r} \lambda F_{\lambda}(r)-e^{-\lambda r} x^{\mu-1 / 2}\left(\mu^{2}-1 / 4\right) \lambda / 2 x \\
& =e^{-\lambda r} x^{\mu-1 / 2} r+\lambda e^{-\lambda r} \int_{0}^{\infty}\left(e^{-r v}-1\right) w_{\lambda}(v) d v .
\end{aligned}
$$

We recall that $Q(r)=r-\left(\mu^{2}-1 / 4\right) \lambda / 2 x$ and $F_{\lambda}(r)=\int_{0}^{\infty} e^{-r v} w_{\lambda}(v) d v$. To simplify the proof we set

$$
w_{\lambda}^{\#}(v)=-\int_{v}^{\infty} w_{\lambda}(\nu) d \nu, \quad \text { that is, } \frac{d w_{\lambda}^{\#}(v)}{d v}=w_{\lambda}(v) .
$$

Then by integration by parts we obtain

$$
\begin{aligned}
\int_{0}^{\infty}\left(e^{-r v}-1\right) w_{\lambda}(v) d v & =\left.\left(e^{-r v}-1\right) w_{\lambda}^{\#}(v)\right|_{0} ^{\infty}+r \int_{0}^{\infty} e^{-r v} w_{\lambda}^{\#}(v) d v \\
& =r \int_{0}^{\infty} e^{-r v} w_{\lambda}^{\#}(v) d v .
\end{aligned}
$$


Thus, the left-hand side of (12) is now of the form

$$
\begin{aligned}
& \int_{0}^{\infty} r x^{\mu} \frac{K_{\mu}(x r)}{K_{\mu}(r)} \cos (r y) / r d r \\
& \quad=x^{\mu-1 / 2} \int_{0}^{\infty} e^{-\lambda r} \cos (r y) d r+\int_{0}^{\infty} \lambda e^{-\lambda r}\left(\int_{0}^{\infty}\left(e^{-r v}-1\right) w_{\lambda}(v) d v\right) \cos (r y) / r d r \\
& =x^{\mu-1 / 2} \int_{0}^{\infty} e^{-\lambda r} \cos (r y) d r+\int_{0}^{\infty} \lambda e^{-\lambda r}\left(\int_{0}^{\infty} e^{-r v} w_{\lambda}^{\#}(v) d v\right) \cos (r y) d r \\
& \quad=H_{\mu}^{(1)}(y)+H_{\mu}^{(2)}(y)=H_{\mu}(y) .
\end{aligned}
$$

Using the standard formula for the Laplace transform of the cosine function we obtain

$$
H_{\mu}^{(1)}(y)=x^{\mu-1 / 2} \frac{\lambda}{\lambda^{2}+y^{2}}, \quad H_{\mu}^{(2)}(y)=\lambda \int_{0}^{\infty} \frac{(\lambda+v) w_{\lambda}^{\#}(v)}{(\lambda+v)^{2}+y^{2}} d v .
$$

Hence by (6) (applied for $s=1$ ), (10) and (11) we obtain

$$
H_{\mu}^{(1)}(y)=\frac{x^{\mu-1 / 2} \lambda}{2^{2}} \int_{0}^{\infty} e^{-y^{2} / 4 t} e^{-\lambda^{2} / 4 t} \frac{d t}{t^{2}} .
$$

Analogously, for $H_{\mu}^{(2)}$ we obtain

$$
\begin{aligned}
H_{\mu}^{(2)}(y) & =\frac{\lambda}{2^{2}} \int_{0}^{\infty} e^{-y^{2} / 4 t}\left(\int_{0}^{\infty}(\lambda+v) w_{\lambda}^{\#}(v) e^{-(\lambda+v)^{2} / 4 t} d v\right) \frac{d t}{t^{2}} \\
& =\frac{\lambda}{2} \int_{0}^{\infty} e^{-y^{2} / 4 t}\left(\int_{0}^{\infty} \frac{d\left(-e^{-(\lambda+v)^{2} / 4 t}\right)}{d v} w_{\lambda}^{\#}(v) d v\right) \frac{d t}{t} .
\end{aligned}
$$

Observe now that

$$
\begin{aligned}
\int_{0}^{\infty} \frac{d\left(-e^{-(\lambda+v)^{2} / 4 t}\right)}{d v} & w_{\lambda}^{\#}(v) d v \\
& =-\left.e^{-(\lambda+v)^{2} / 4 t} w_{\lambda}^{\#}(v)\right|_{0} ^{\infty}+\int_{0}^{\infty} e^{-(\lambda+v)^{2} / 4 t} \frac{d w_{\lambda}^{\#}(v)}{d v} d v \\
& =e^{-\lambda^{2} / 4 t} w_{\lambda}^{\#}(0)+\int_{0}^{\infty} e^{-(\lambda+v)^{2} / 4 t} w_{\lambda}(v) d v
\end{aligned}
$$

We also have

$$
w_{\lambda}^{\#}(0)=-\int_{0}^{\infty} \frac{d w_{\lambda}^{\#}(v)}{d v} d v=-\int_{0}^{\infty} w_{\lambda}(v) d v
$$


By the above identities and the form of $H_{\mu}^{(2)}$ we obtain

$$
H_{\mu}^{(2)}(y)=\frac{\lambda}{2} \int_{0}^{\infty} e^{-y^{2} / 4 t}\left(\int_{0}^{\infty}\left(e^{-\kappa / 4 t}-1\right) w_{\lambda}(v) d v\right) \frac{d t}{t}
$$

Combining the above identities we conclude that the left-hand side of (12) takes the form

$$
H_{\mu}(y)=\frac{\lambda}{2} \int_{0}^{\infty} e^{-y^{2} / 4 t} e^{-\lambda^{2} / 4 t}\left(x^{\mu-1 / 2} / 2 t+\int_{0}^{\infty}\left(e^{-\kappa / 4 t}-1\right) w_{\lambda}(v) d v\right) \frac{d t}{t} .
$$

Taking into account the right-hand side of (12) and continuity of $q_{\mu}$ with respect to $t$ (see properties of $w_{\lambda}$ below), we obtain (13). When $\mu>1 / 2$, then using (11) we obtain (14).

Below we give a description of the function $w_{\lambda}$. We rely on the results of [BGS]. The formulas depend on the zeros of $K_{\mu}(z)$.

Even if in general these zeros are not given explicitly, we are able to prove some important properties (like boundedness or asymptotics) of $w_{\lambda}$, which are essential in applications. Moreover, for some values of $\mu$ we do provide explicit formulas (see Corollary 3.5).

The function $z^{\mu} K_{\mu}(z)$ extends to an entire function when $\mu-1 / 2$ is an integer, and has a holomorphic extension to $\mathbb{C} \backslash(-\infty, 0]$ when $\mu-1 / 2$ is not an integer. Denote the set of zeros of $K_{\mu}(z)$ by $Z=\left\{z_{1}, \ldots, z_{k_{\mu}}\right\}$. We recall some information about these zeros (cf. [E, p. 62]). We have $k_{\mu}=\mu-1 / 2$ when $\mu-1 / 2 \in \mathbb{N}$. For $\mu-1 / 2 \notin \mathbb{N}, k_{\mu}$ is the even number closest to $\mu-1 / 2$. In particular, $k_{\mu}=0$ for $0 \leq \mu<3 / 2$, and $k_{\mu}=2$ for $\mu=2$ and 3 . The functions $K_{\mu}$ and $K_{\mu-1}$ have no common zeros.

As in [BGS], we need an additional notation to describe the function $w_{\lambda}$. For $\mu>0$ define

$$
w_{1, \lambda}(v)=-\frac{x^{\mu}}{\lambda} \sum_{i=1}^{k_{\mu}} \frac{z_{i} e^{\lambda z_{i}} K_{\mu}\left(x z_{i}\right)}{K_{\mu-1}\left(z_{i}\right)} e^{z_{i} v} .
$$

When $\mu+1 / 2 \notin \mathbb{N}$ and $\mu \geq 0$ we define

$$
\begin{aligned}
w_{2, \lambda}(v) & =-\cos (\pi \mu) \frac{x^{\mu}}{\lambda} \\
\times & \int_{0}^{\infty} \frac{I_{\mu}(x u) K_{\mu}(u)-I_{\mu}(u) K_{\mu}(x u)}{\cos ^{2}(\pi \mu) K_{\mu}^{2}(u)+\left(\pi I_{\mu}(u)+\sin (\pi \mu) K_{\mu}(u)\right)^{2}} e^{-\lambda u} e^{-v u} u d u .
\end{aligned}
$$

We now formulate our representation theorem for the function $w_{\lambda}$. The proof of the main part is the same as in [BGS] and is omitted; we only show the asymptotic properties of $w_{2, \lambda}$. For $\mu=0$ this is new; the behaviour for $\mu=(n-1) / 2$ was shown in [BGS]. Nevertheless, we present a new and unified proof based on Tauberian theorems. 
Theorem 3.4. Let $\lambda=x-1>0$. In the case $\mu-1 / 2 \in \mathbb{N}$,

$$
w_{\lambda}(v)=w_{1, \lambda}(v)
$$

while in the case $\mu-1 / 2 \notin \mathbb{N}$,

$$
w_{\lambda}(v)=w_{1, \lambda}(v)+w_{2, \lambda}(v) .
$$

Moreover, $\sup _{v \geq 0}\left|w_{\lambda}(v)\right|<\infty$, and

$$
\begin{gathered}
-\cos (\pi \mu) w_{2, \lambda}(v) \geq 0, \quad v \geq 0 \quad(\mu-1 / 2 \notin \mathbb{N}) ; \\
\int_{0}^{\infty} v^{k}\left|w_{1, \lambda}(v)\right| d v<\infty, \quad k=1,2, \ldots ; \\
\lim _{v \rightarrow \infty} v^{k} w_{1, \lambda}(v)=0, \quad k=1,2, \ldots ; \\
\lim _{v \rightarrow \infty} v^{2 \mu+2} w_{2, \lambda}(v)=\frac{-\cos (\pi \mu) \Gamma(2 \mu+2)}{2^{2 \mu-3} \Gamma(\mu) \Gamma(\mu+1)} \frac{x^{2 \mu}-1}{\lambda} \quad(\mu-1 / 2 \notin \mathbb{N}, \mu>0) ; \\
\lim _{v \rightarrow \infty}(v \log v)^{2} w_{2, \lambda}(v)=-\frac{1}{2 \lambda} \log x \quad \text { for } \mu=0 .
\end{gathered}
$$

Proof. Define

$$
h_{\mu, \lambda}(u)=\frac{I_{\mu}(x u) K_{\mu}(u)-I_{\mu}(u) K_{\mu}(x u)}{\cos ^{2}(\pi \mu) K_{\mu}^{2}(u)+\left(\pi I_{\mu}(u)+\sin (\pi \mu) K_{\mu}(u)\right)^{2}} e^{-\lambda u} u .
$$

Observe that the function $h_{\mu, \lambda}(u)$ is non-negative $\left(I_{\mu}(u) / K_{\mu}(u)\right.$ is increasing for $u>0)$ and $w_{2, \lambda}(v)$ is the Laplace transform of $-\cos (\pi \mu) \frac{x^{\mu}}{\lambda} h_{\mu, \lambda}$ at $v$. We claim that for $u \rightarrow 0+$ we have the following asymptotics for $h_{\mu, \lambda}$ :

$$
h_{\mu, \lambda}(u) \approx \begin{cases}x^{\mu} \frac{c_{\mu}}{c_{\mu}^{\prime}}\left(1-x^{-2 \mu}\right) u^{2 \mu+1} & \text { for } \mu>0, \\ u \log x(\log u)^{-2} & \text { for } \mu=0 .\end{cases}
$$

Applying Karamata's Tauberian theorem (see, e.g., [Fe, Ch. XIII, 5, p. 422]) we find that

$$
\lim _{v \rightarrow \infty} w_{2, \lambda}(v)\left(\int_{0}^{1 / v} h_{\mu, \lambda}(u) d u\right)^{-1}=\frac{-\cos (\pi \mu)}{\lambda} \Gamma(2+2 \mu) x^{\mu} .
$$

This together with (18) implies

$$
\begin{gathered}
\lim _{v \rightarrow \infty} v^{2 \mu+2} w_{2, \lambda}(v)=\frac{-\cos (\pi \mu) \Gamma(2 \mu+2)}{2^{2 \mu-3} \Gamma(\mu) \Gamma(\mu+1)} \frac{x^{2 \mu}-1}{\lambda} \quad(\mu-1 / 2 \notin \mathbb{N}, \mu>0), \\
\lim _{v \rightarrow \infty}(v \log v)^{2} w_{2, \lambda}(v)=-\frac{1}{2 \lambda} \log x, \quad \mu=0 .
\end{gathered}
$$

To prove (18) we apply the following asymptotics. When $u \rightarrow 0$ we have:

$$
\begin{array}{rlrl}
I_{\mu}(u) & \approx c_{\mu} u^{\mu}, & K_{\mu}(u) & \approx c_{\mu}^{\prime} u^{-\mu} ; \\
I_{0}(u) & =1+o(1), & K_{0}(u)=\log (2 / u) I_{0}(u)+\psi(1)+o(1),
\end{array}
$$


with $c_{\mu}=2^{-\mu} / \Gamma(\mu+1), c_{\mu}^{\prime}=2^{\mu-1} \Gamma(\mu)$ and where $\psi$ is the Euler function. Then

$$
\begin{aligned}
e^{\lambda u} u^{-1} h_{\mu, \lambda}(u) & \frac{I_{\mu}(x u) K_{\mu}(u)}{K_{\mu}^{2}(u)+\left(\pi I_{\mu}(u)\right)^{2}+2 \pi \sin (\pi \mu) K_{\mu}(u) I_{\mu}(u)}\left(1-\frac{I_{\mu}(u) K_{\mu}(x u)}{I_{\mu}(x u) K_{\mu}(u)}\right) \\
& \approx \frac{I_{\mu}(x u)}{K_{\mu}(u)}\left(1-\frac{I_{\mu}(u) K_{\mu}(x u)}{I_{\mu}(x u) K_{\mu}(u)}\right) \\
& \approx \begin{cases}u^{2 \mu} x^{\mu} \frac{c_{\mu}}{c_{\mu}^{\prime}}\left(1-x^{-2 \mu}\right) & \text { for } \mu>0, \\
\frac{\log x}{(\log u)^{2}} & \text { for } \mu=0 .\end{cases}
\end{aligned}
$$

ExAmples. To illustrate the representation theory developed so far we write down explicit integral formulas for the density $q_{\mu}$ in some special cases of $\mu$. All these formulas follow directly from Theorems 3.3 and 3.4. If $0 \leq \mu<$ $3 / 2$ then $w_{\lambda}=w_{2, \lambda}$ and the functions $w_{\lambda}$ have constant sign. For $\mu=0$ and $\mu=1$ the function $w_{\lambda}$ has a simpler form, which we exhibit here. If $\mu=1 / 2$ then $F_{\lambda}=0$ and $q_{\mu}(t)$ reduces to the standard $1 / 2$-stable subordinator. For $\mu+1 / 2 \in \mathbb{N}$ we have $w_{\lambda}=w_{1, \lambda}$, and $w_{1, \lambda}$ can be computed by calculating residues of simple rational functions (see the formula for $w_{1, \lambda}$ or calculations in [BGS]). Again, we give the explicit form of $w_{\lambda}$ for $\mu=3 / 2$ and $\mu=5 / 2$.

Corollary 3.5. Let $\kappa=\kappa(v)=(\lambda+v)^{2}-\lambda^{2}=v(2 \lambda+v)$, where $\lambda=x-1>0$. If $\mu=0$ then

$$
\begin{aligned}
-w_{\lambda}(v) & =\frac{1}{\lambda} \int_{0}^{\infty} \frac{I_{0}(x u) K_{0}(u)-K_{0}(x u) I_{0}(u)}{K_{0}^{2}(u)+\pi^{2} I_{0}^{2}(u)} e^{-u \lambda} e^{-v u} u d u, \\
q_{\mu}(t) & =\lambda \frac{e^{-\lambda^{2} / 4 t}}{\sqrt{\pi t}}\left((\lambda+1)^{-1 / 2} / 2 t+\int_{0}^{\infty}\left(1-e^{-\kappa / 4 t}\right)\left(-w_{\lambda}(v)\right) d v\right) .
\end{aligned}
$$

If $\mu=1 / 2$ then

$$
q_{\mu}(t)=\lambda \frac{e^{-\lambda^{2} / 4 t}}{2 \sqrt{\pi t^{3}}} .
$$

If $\mu=1$ then

$$
\begin{aligned}
w_{\lambda}(v) & =\frac{\lambda+1}{\lambda} \int_{0}^{\infty} \frac{I_{1}(x u) K_{1}(u)-K_{1}(x u) I_{1}(u)}{K_{1}^{2}(u)+\pi^{2} I_{1}^{2}(u)} e^{-u \lambda} e^{-v u} u d u \\
q_{\mu}(t) & =\lambda \frac{e^{-\lambda^{2} / 4 t}}{\sqrt{\pi t}} \int_{0}^{\infty}\left(e^{-\kappa / 4 t}-1+\kappa / 4 t\right) w_{\lambda}(v) d v
\end{aligned}
$$




$$
\begin{aligned}
& \text { If } \mu=3 / 2 \text { then } w_{\lambda}(v)=e^{-v} \text { and } \\
& \qquad q_{\mu}(t)=\lambda \frac{e^{-\lambda^{2} / 4 t}}{\sqrt{\pi t}} \int_{0}^{\infty}\left(e^{-\kappa / 4 t}-1+\kappa / 4 t\right) e^{-v} d v .
\end{aligned}
$$

If $\mu=5 / 2$ then

$$
\begin{aligned}
& w_{\lambda}(v)=3 e^{-3 v / 2}[(2 \lambda+1) \cos (\sqrt{3} v / 2)+\sqrt{3} \sin (\sqrt{3} v / 2)], \\
& q_{\mu}(t)=\lambda \frac{e^{-\lambda^{2} / 4 t}}{\sqrt{\pi t}} \int_{0}^{\infty}\left(e^{-\kappa / 4 t}-1+\kappa / 4 t\right) w_{\lambda}(v) d v .
\end{aligned}
$$

4. Asymptotic behaviour of $A(\tau)$. In this section we prove the following

THEOREM 4.1. The density $q_{\mu}$ of $A(\tau)$ satisfies:

$$
\begin{aligned}
\lim _{t \rightarrow \infty} t^{\mu+1} q_{\mu}(t) & =C_{\mu} \quad \text { if } \mu>0, \\
\lim _{t \rightarrow \infty}(\log t)^{2} t q_{\mu}(t) & =C_{0} \quad \text { if } \mu=0,
\end{aligned}
$$

for some positive $C_{\mu}$.

The above theorem provides the following important information about integrability of $A(\tau)$ :

COROllary 4.2. For $\mu>0$ the following holds:

$$
E A^{p}(\tau)<\infty \text { if and only if } p<\mu .
$$

REMARK. The above corollary can be stated equivalently in terms of Bessel processes as follows: the first hitting time of level 1 for a Bessel process with index $-\mu, \mu>0$, starting at $x>1$, has finite positive moments of order $p$ if and only if $p<\mu$.

The proof of Theorem 4.1 is contained in a series of lemmas.

Lemma 4.3. Let $\mu>0$. There exists a constant $C>1$ such that

$$
C^{-1} t^{-\mu} \leq P(A(\tau)>t) \leq C t^{-\mu}, \quad t>1 .
$$

Proof. We write the basic formula (2) for the case we work with, that is, when $x>1$ and $a=1$ :

$$
A(\infty)=A(\tau)+x^{-2} A^{\prime}(\infty) .
$$

We recall that $A(\infty), A(\tau)$ denote $A_{x}(\infty), A_{x}\left(\tau_{1}\right)$, respectively, and, as before, $A^{\prime}(\infty)$ is a copy of $A(\infty)$, independent of $A(\tau)$. Applying the above formula we obtain

$$
P(A(\infty)>t) \leq P\left(A(\tau)>\left(1-x^{-1}\right) t\right)+P(A(\infty)>x t),
$$

which implies

$$
P(t \leq A(\infty) \leq x t) \leq P\left(A(\tau)>\left(1-x^{-1}\right) t\right) .
$$


Moreover,

$$
P(A(\tau)>t) \leq P(A(\infty)>t) .
$$

Now the lemma follows from $(21)$ and $(22)$ since $P(A(\infty)>t) \approx c t^{-\mu}$ for some $c>0$ by $(3)$.

Recall that $\kappa=\kappa(v)=(\lambda+v)^{2}-\lambda^{2}$, where $\lambda=x-1>0$.

LEMMA 4.4. Let $m \in \mathbb{N}$ be such that $2 \leq m \leq \mu+1 / 2$. Then

$$
\begin{array}{r}
\lim _{t \rightarrow \infty} t^{m} \int_{0}^{\infty} w_{\lambda}(v)\left(e^{-\kappa / 4 t}-\sum_{0 \leq j \leq m-1}(-1)^{j} \frac{1}{j !}\left(\frac{\kappa}{4 t}\right)^{j}\right) d v \\
=\frac{(-1)^{m}}{4^{m} m !} \int_{0}^{\infty} \kappa^{m} w_{\lambda}(v) d v=C_{m} .
\end{array}
$$

Moreover, $C_{m}=0$ for $2 \leq m<\mu+1 / 2$, and $C_{m}>0$ if $m=\mu+1 / 2 \in \mathbb{N}$.

Proof. Define

$$
\psi(t, m)=t^{m} w_{\lambda}(v)\left(e^{-\kappa / 4 t}-\sum_{0 \leq j \leq m-1}(-1)^{j} \frac{1}{j !}\left(\frac{\kappa}{4 t}\right)^{j}\right) .
$$

By elementary calculations,

$$
\left|e^{-\kappa / 4 t}-\sum_{0 \leq j \leq m-1}(-1)^{j} \frac{1}{j !}\left(\frac{\kappa}{4 t}\right)^{j}\right| \leq \frac{1}{m !}\left(\frac{\kappa}{4 t}\right)^{m} .
$$

Hence

$$
|\psi(t, m)| \leq\left|w_{\lambda}(v)\right| \frac{\kappa^{m}}{m !}
$$

Under the assumption on $m$ the function $\left|w_{\lambda}(v)\right| \kappa^{m}$ is integrable so the formula (23) follows from the bounded convergence theorem.

Suppose that $C_{m} \neq 0$ for some $m$ with $2 \leq m \leq \mu+1 / 2$, and set

$$
m_{0}=\inf \left\{m \in \mathbb{N}: 2 \leq m \leq \mu+1 / 2, C_{m} \neq 0\right\} \text {. }
$$

Then from the first part of the proof we have

$$
\lim _{t \rightarrow \infty} t^{m_{0}+1 / 2} q_{\mu}(t)=C_{m_{0}}>0
$$

which implies that

$$
\lim _{t \rightarrow \infty} t^{m_{0}-1 / 2} P(A(\tau)>t)=C_{m_{0}}\left(m_{0}-1 / 2\right)^{-1} .
$$

From Lemma 4.3 we infer that $C_{m_{0}}>0$ if and only if $m_{0}-1 / 2=\mu$. In particular, we then have $\mu+1 / 2 \in \mathbb{N}$, and $C_{m}=0$ if $m<\mu+1 / 2$.

REMARK. The above lemma shows, in particular, that for $m \in \mathbb{N}, 2 \leq$ $m<\mu+1 / 2$,

$$
\int_{0}^{\infty} \kappa^{m} w_{\lambda}(v) d v=0
$$


Thus, the representation formula for the density $q_{\mu}$ can be written for $\mu \geq$ $1 / 2$ as follows:

$$
q_{\mu}(t)=\lambda \frac{e^{-\lambda^{2} / 4 t}}{\sqrt{\pi t}} \int_{0}^{\infty}\left(e^{-\kappa / 4 t}-\sum_{0 \leq j \leq l}(-1)^{j} \frac{1}{j !}\left(\frac{\kappa}{4 t}\right)^{j}\right) w_{\lambda}(v) d v,
$$

where $l=[\mu+1 / 2]$ if $\mu-1 / 2 \notin \mathbb{N}$, and $l=\mu-1 / 2$ otherwise.

We now prove our theorem.

Proof of Theorem 4.1. For $\mu=1 / 2$ the density $q_{\mu}$ has a particularly simple form (see Corollary 3.5) and the theorem clearly holds true. Hence, we assume throughout the remainder of the proof that $\mu \neq 1 / 2$. Next, if $\mu-1 / 2 \in \mathbb{N}$ then the remark above together with Lemma 4.4 yield our theorem at once.

Thus, we assume that $\mu-1 / 2 \notin \mathbb{N}$ and let $l=[\mu+1 / 2]$. Define

$$
I(t)=\int_{0}^{\infty} w_{\lambda}(v) t^{\mu+1 / 2}\left(e^{-\kappa / 4 t}-\sum_{0 \leq j \leq l}(-1)^{j} \frac{1}{j !}\left(\frac{\kappa}{4 t}\right)^{j}\right) d v .
$$

We prove that

$$
\begin{aligned}
\lim _{t \rightarrow \infty} I(t)=C>0 & \text { if } \mu>0, \\
\lim _{t \rightarrow \infty}(\log t)^{2} I(t)=C>0 & \text { if } \mu=0 .
\end{aligned}
$$

Applying the change of variable $\kappa=4 s t$ we obtain

so

$$
v=\sqrt{4 s t+\lambda^{2}}-\lambda, \quad d v=\frac{2 t}{\sqrt{4 s t+\lambda^{2}}} d s,
$$

where

$$
I(t)=\int_{0}^{\infty} \psi_{\lambda}(s, t)\left(e^{-s}-\sum_{0 \leq j \leq l}(-1)^{j} \frac{s^{j}}{j !}\right) d s,
$$

$$
\psi_{\lambda}(s, t)=w_{\lambda}\left(\sqrt{4 s t+\lambda^{2}}-\lambda\right) \frac{2 t^{\mu+3 / 2}}{\sqrt{4 s t+\lambda^{2}}} .
$$

We claim that for $\mu>0$ and $t, s \in \mathbb{R}^{+}$there is a constant $C>0$ such that

$$
\left|\psi_{\lambda}(s, t)\right| \leq C s^{-\mu-3 / 2},
$$

and for $\mu=0$ and $t, s \in \mathbb{R}^{+}$,

$$
(\log t)^{2}\left|\psi_{\lambda}(s, t)\right| \leq C \max \left\{1,(\log s)^{2}\right\} s^{-3 / 2} .
$$

The above claims prove the relations (25) and (26). Indeed, consider first the case $\mu>0$. Then the absolute value of the integrand in $I(t)$ can be estimated by the integrable function

$$
C\left|e^{-s}-\sum_{0 \leq j \leq l}(-1)^{j} \frac{s^{j}}{j !}\right| s^{-\mu-3 / 2} \leq C \min \{1, s\} s^{l} s^{-\mu-3 / 2}=C \min \{1, s\} s^{-\delta-1},
$$


where $\delta=\mu+1 / 2-[\mu+1 / 2], 0<\delta<1$. Then the proof of (25) is concluded by letting $t \rightarrow \infty$ and using the asymptotics of $w_{\lambda}$ (see Theorem 3.4).

Now, consider the case $\mu=0$. Observe that the absolute value of the integrand in $(\log t)^{2} I(t)$ is estimated by the integrable function

$$
C \max \left\{1,(\log s)^{2}\right\} s^{-3 / 2}\left|e^{-s}-1\right|,
$$

So

$$
\lim _{t \rightarrow \infty}(\log t)^{2} I(t)=\frac{\log x}{2 \lambda} \int_{0}^{\infty}\left(1-e^{-s}\right) s^{-3 / 2} d s .
$$

Here we take into account

$$
(\log t)^{2} \psi_{\lambda}(s, t) \approx-\frac{\log x}{2 \lambda} \frac{(\log t)^{2}}{s^{3 / 2}(\log s t)^{2}} \approx-\frac{\log x}{2 \lambda s^{3 / 2}}, \quad t \rightarrow \infty
$$

by using the asymptotics of $w_{\lambda}$ (see Theorem 3.4 ).

Now we can conclude the proof of the asymptotic behaviour of $q_{\mu}(t)$ in the case $\mu-1 / 2 \notin \mathbb{N}$. Note that for $0<\mu<1 / 2$ we have

$$
\begin{aligned}
t^{\mu+1} q_{\mu}(t) & =\lambda \frac{e^{-\lambda^{2} / 4 t}}{\sqrt{\pi t}}\left(t^{\mu} x^{\mu-1 / 2} / 2+t^{\mu+1} \int_{0}^{\infty}\left(e^{-\kappa / 4 t}-1\right) w_{\lambda}(v) d v\right) \\
& =\lambda \frac{e^{-\lambda^{2} / 4 t}}{\sqrt{\pi}}\left(t^{\mu-1 / 2} x^{\mu-1 / 2} / 2+I(t)\right) \rightarrow \lambda C / \sqrt{\pi},
\end{aligned}
$$

where $C$ is the constant from (25). The same argument for $\mu=0$ shows that

$$
(\log t)^{2} t q_{\mu}(t) \rightarrow \lambda C / \sqrt{\pi},
$$

where $C$ comes from (26). For $\mu>1 / 2$ the asymptotics of $q_{\mu}(t)$ follows directly from (25).

We now justify our claims (27) and (28). We use the notation introduced in the proof of the theorem.

LEMMA 4.5. If $\mu>0$ there is $c>0$ such that

$$
\left|\psi_{\lambda}(s, t)\right| \leq c s^{-\mu-3 / 2} \text {. }
$$

For $\mu=0$ there is $C>0$ such that

$$
(\log t)^{2}\left|\psi_{\lambda}(s, t)\right| \leq C \max \left\{1,(\log s)^{2}\right\} s^{-3 / 2} .
$$

Proof. Let $\mu>0$. Since $w_{\lambda}$ is bounded, for $t s \leq 1$ we have

$$
\left|\psi_{\lambda}(s, t)\right| \leq \sup _{v \geq 0}\left|w_{\lambda}(v)\right| \frac{t^{\mu+3 / 2}}{\sqrt{4 s t+\lambda^{2}}} \leq C s^{-\mu-3 / 2} .
$$

For $t s \geq 1$ we use the asymptotics of $w_{\lambda}$ at $\infty$ (see Theorem 3.4 ) to arrive at

$$
\left|\psi_{\lambda}(s, t)\right| \leq C t^{\mu+3 / 2}(s t)^{-\mu-3 / 2}=C s^{-\mu-3 / 2} .
$$


For $\mu=0$, again using the boundedness of $w_{\lambda}$ we estimate for $t s \leq 2$ :

$$
\left|\psi_{\lambda}(s, t)\right| \leq \sup _{v \geq 0}\left|w_{\lambda}(v)\right| \frac{t^{3 / 2}}{\sqrt{4 s t+\lambda^{2}}} \leq C s^{-3 / 2} .
$$

For $t s \geq 2$ we use the asymptotics of $w_{\lambda}$ to get

$$
(\log t)^{2}\left|\psi_{\lambda}(s, t)\right| \leq C t^{3 / 2}(s t)^{-3 / 2} \frac{(\log t)^{2}}{(\log s t)^{2}} \leq \begin{cases}C s^{-3 / 2}(\log t)^{2}, & 2 \leq t s \leq \sqrt{t}, \\ C s^{-3 / 2}, & t s \geq \sqrt{t}\end{cases}
$$

Next observe that for $2 \leq t s \leq \sqrt{t}$ we have $(\log t)^{2} \leq 4(\log s)^{2}$.

5. Hyperbolic Brownian motion with drift. Consider the half-space model of the $n$-dimensional real hyperbolic space

$$
\mathbb{H}^{n}=\left\{\left(x_{1}, \ldots, x_{n-1}, x_{n}\right) \in \mathbb{R}^{n-1} \times \mathbb{R}: x_{n}>0\right\} .
$$

The Riemannian metric, the volume element and the Laplace-Beltrami operator are given by

$$
\begin{aligned}
d s^{2} & =\frac{d x_{1}^{2}+\cdots+d x_{n-1}^{2}+d x_{n}^{2}}{x_{n}^{2}}, \\
d V & =\frac{d x_{1} \cdots d x_{n-1} d x_{n}}{x_{n}^{n}}, \\
\Delta & =x_{n}^{2} \sum_{i=1}^{n} \partial_{i}^{2}-(n-2) x_{n} \partial_{n},
\end{aligned}
$$

respectively (here $\partial_{i}=\partial / \partial x_{i}, i=1, \ldots, n$ ). For $\mu \geq 0$ let $\alpha=2 \mu-n+1$. We also introduce the operator

$$
\Delta_{\mu}=\Delta-\alpha x_{n} \partial_{n}=x_{n}^{2} \sum_{i=1}^{n} \partial_{i}^{2}-(2 \mu-1) x_{n} \partial_{n} .
$$

Let $\left(B_{i}(t)\right)_{i=1, \ldots, n}$ be a family of independent classical Brownian motions on $\mathbb{R}$ with the generator $\frac{d^{2}}{d x^{2}}$ (and not $\frac{1}{2} \frac{d^{2}}{d x^{2}}$ ), i.e. $E^{0} B_{i}^{2}(t)=2 t$. Then the Brownian motion on $\mathbb{H}^{n}, X=\left(X_{i}\right)_{i=1, \ldots, n}$, can be described by the following system of stochastic differential equations:

$$
\left\{\begin{aligned}
d X_{1}(t) & =X_{n}(t) d B_{1}(t) \\
d X_{2}(t) & =X_{n}(t) d B_{2}(t) \\
\ldots \ldots \ldots \ldots \ldots \ldots \ldots \ldots \ldots \ldots \ldots \ldots \ldots \ldots \ldots & \ldots \ldots \ldots \\
d X_{n}(t) & =X_{n}(t) d B_{n}(t)-(n-2) X_{n}(t) d t .
\end{aligned}\right.
$$

More generally, if we replace $n-2$ by $\alpha+n-2=2 \mu-1$ then the corresponding process will be called the Brownian motion on $\mathbb{H}^{n}$ with drift $\alpha$. By using the Itô formula one verifies that the generator of the solution of this system 
is $\Delta_{\mu}$. Moreover, it can be easily checked that the solution is given by

$$
\left\{\begin{array}{c}
X_{1}(t)=X_{1}(0)+\int_{0}^{t} X_{n}(t) d B_{1}(s), \\
X_{2}(t)=X_{2}(0)+\int_{0}^{t} X_{n}(t) d B_{2}(s), \\
\ldots \ldots \ldots \ldots \ldots \ldots \ldots \ldots \ldots \ldots \ldots \ldots \ldots \\
X_{n}(t)=X_{n}(0) \exp \left(B_{n}(t)-2 \mu t\right) .
\end{array}\right.
$$

Define the projection $\sim$ : $\mathbb{R}^{n} \ni u=\left(u_{1}, \ldots, u_{n}\right) \mapsto \widetilde{u}=\left(u_{1}, \ldots, u_{n-1}\right) \in \mathbb{R}^{n-1}$. In particular, $\widetilde{X}(t)=\left(X_{1}(t), \ldots, X_{n-1}(t)\right)$. From the representation above one may easily verify (e.g. by computing Fourier transforms) that

$$
\widetilde{X}(t) \stackrel{d}{=} \widetilde{X}(0)+\widetilde{B}\left(\int_{0}^{t} X_{n}^{2}(s) d s\right), \quad t \geq 0,
$$

where $\widetilde{B}(t)=\left(B_{1}(t), \ldots, B_{n-1}(t)\right)$ is an $(n-1)$-dimensional Brownian motion independent of the process $X_{n}(t)$. Consider a half-space $D=\left\{u \in \mathbb{H}^{n}\right.$ : $\left.u_{n}>a\right\}$ for some fixed $a>0$. To simplify the notation we choose $a=1$. Define

$$
\tau=\inf \{t \geq 0: X(t) \notin D\}=\inf \left\{t \geq 0: X_{n}(t)=1\right\} .
$$

We denote by $P_{1}(u, y), u=\left(u_{1}, \ldots, u_{n}\right) \in D, y=\left(y_{1}, \ldots, y_{n-1}, 1\right) \in \partial D$ the Poisson kernel of $D$, i.e. the density of the distribution of $X(\tau)$ starting at $u$ (since $X_{n}(\tau)=1$ it is enough to consider the distribution of $\widetilde{X}(\tau)$ ). From (29) it is obvious that

$$
\widetilde{X}(\tau) \stackrel{d}{=} \widetilde{u}+\widetilde{B}(A(\tau)),
$$

where the functional $A(\tau)$ (starting from $u_{n}>1$ ) is independent of $\widetilde{B}(t)$. For further considerations we may take $\widetilde{u}=0$ and $u_{n}=x>1$, so the starting point of the $n$-dimensional process $X(\cdot)$ is $(0, \ldots, 0, x) \in D$. Since $A(\tau)$ and $\widetilde{B}(t)$ are independent, we have the following representation of the Poisson kernel:

Corollary 5.1.

$$
P_{1}(x, y)=\frac{1}{(4 \pi)^{(n-1) / 2}} \int_{0}^{\infty} e^{-|y|^{2} / 4 t} q_{\mu}(t) \frac{d t}{t^{(n-1) / 2}} .
$$

Observe that for $\mu=1 / 2$ the functional $A(\tau)$ has the standard asymmetric $1 / 2$-stable distribution and the Poisson kernel is exactly the $(n-1)$ dimensional Cauchy density, so in what follows, we consider $\mu \neq 1 / 2$.

Taking into account the formula (3) and Lemma 2.1 we obtain the following representation of the Poisson kernel of the set $D$ : 
Theorem 5.2. Set $\varrho=|y|$. Let $\kappa=\kappa(v)=(\lambda+v)^{2}-\lambda^{2}$, where $\lambda=$ $x-1>0$. For $0 \leq \mu<1 / 2$ we have

$$
\begin{aligned}
P_{1}(x, y)= & \frac{\Gamma(n / 2-1)}{2 \pi^{n / 2}} \frac{\lambda}{\left(\lambda^{2}+\varrho^{2}\right)^{n / 2}} \\
& \times\left[(n-2)(\lambda+1)^{\mu-1 / 2}-\int_{0}^{\infty} \frac{w_{\lambda}(v) L^{\#}(\lambda, \varrho, v) d v}{\left((\lambda+v)^{2}+\varrho^{2}\right)^{n / 2-1}}\right],
\end{aligned}
$$

where $L^{\#}(\lambda, \varrho, v)$ is defined by

$$
\frac{L^{\#}(\lambda, \varrho, v)}{\left(\lambda^{2}+\varrho^{2}\right)\left((\lambda+v)^{2}+\varrho^{2}\right)^{n / 2-1}}=1-\left(\frac{\lambda^{2}+\varrho^{2}}{(\lambda+v)^{2}+\varrho^{2}}\right)^{n / 2-1} .
$$

For $\mu>1 / 2$ we obtain

$$
P_{1}(x, y)=\frac{\Gamma(n / 2-1)}{2 \pi^{n / 2}} \frac{\lambda}{\left(\lambda^{2}+\varrho^{2}\right)^{n / 2}} \int_{0}^{\infty} \frac{w_{\lambda}(v) L(\lambda, \varrho, v) d v}{\left((\lambda+v)^{2}+\varrho^{2}\right)^{n / 2-1}},
$$

with $L(\lambda, \varrho, v)$ defined by

$$
\begin{aligned}
& \frac{L(\lambda, \varrho, v)}{\left(\lambda^{2}+\varrho^{2}\right)\left((\lambda+v)^{2}+\varrho^{2}\right)^{n / 2-1}} \\
& =\left(\frac{\lambda^{2}+\varrho^{2}}{(\lambda+v)^{2}+\varrho^{2}}\right)^{n / 2-1}-1+\left(\frac{n}{2}-1\right) \frac{v(2 \lambda+v)}{\lambda^{2}+\varrho^{2}} .
\end{aligned}
$$

Proof. By (6) we obtain

$$
\int_{0}^{\infty} e^{-\varrho^{2} / 4 t} e^{-\lambda^{2} / 4 t} \frac{d t}{t^{1+s}}=\frac{2^{2 s} \Gamma(s)}{\left(\lambda^{2}+\varrho^{2}\right)^{s}} .
$$

Applying the above formula (with different constants) to the three terms appearing in the representation of $q_{\mu}$ we obtain

$$
\begin{aligned}
\int_{0}^{\infty} e^{-\varrho^{2} / 4 t} e^{-\lambda^{2} / 4 t} \frac{d t}{t^{1+n / 2}} & =\frac{2^{n} \Gamma(n / 2)}{\left(\lambda^{2}+\varrho^{2}\right)^{n / 2}}, \\
\int_{0}^{\infty} e^{-\varrho^{2} / 4 t} e^{-\lambda^{2} / 4 t} e^{-\kappa / 4 t} \frac{d t}{t^{n / 2}} & =\frac{2^{n-2} \Gamma(n / 2-1)}{\left((\lambda+v)^{2}+\varrho^{2}\right)^{n / 2-1}}, \\
\int_{0}^{\infty} e^{-\varrho^{2} / 4 t} e^{-\lambda^{2} / 4 t} \frac{d t}{t^{n / 2}} & =\frac{2^{n-2} \Gamma(n / 2-1)}{\left(\lambda^{2}+\varrho^{2}\right)^{n / 2-1}} .
\end{aligned}
$$

Taking into account appropriate constants, we obtain the formulas for the Poisson kernel. 
ThEOREM 5.3.

$$
\begin{aligned}
\lim _{|y| \rightarrow \infty}|y|^{n+2 \mu-1} P_{1}(x, y) & =c_{\mu}, \quad \mu>0, \\
\lim _{|y| \rightarrow \infty}(\log |y|)^{2}|y|^{n-1} P_{1}(x, y) & =c_{0}, \quad \mu=0,
\end{aligned}
$$

for some positive $c_{\mu}$.

Proof. From Corollary 5.1, Theorem 4.1 and arguments presented in its proof (boundedness of $t^{\mu+1} q_{\mu}(t)$ for $\mu>0$ and of $(\log t)^{2} q_{\mu}(t)$ for $\mu=0$ ) we deduce that $P_{1}(x, y)$ has the same asymptotic behaviour (up to a positive constant) as

$$
\int_{0}^{\infty} t^{-1-\mu-(n-1) / 2} e^{-|y|^{2} / 4 t} d t=2^{2 \mu+n-1}|y|^{-2 \mu-n+1} \int_{0}^{\infty} u^{\mu+(n-3) / 2} e^{-u} d u
$$

for $\mu>0$, and similarly for $\mu=0$.

Acknowledgements. We are grateful to the referee, whose remarks and comments allowed us to improve the presentation of the paper. We would also like to thank T. Żak for stimulating conversations on the subject.

\section{References}

[AG] L. Alili and J.-C. Gruet, An explanation of a generalized Bougerol's identity in terms of hyperbolic Brownian motion, in [Y3], pp. 15-33.

[BCF] P. Baldi and E. Casadio Tarabusi, A. Figà-Talamanca, Stable laws arising from hitting distributions of processes on homogeneous trees and the hyperbolic halfplane, Pacific J. Math. 197 (2001), 257-273.

[BCFY] P. Baldi, E. Casadio Tarabusi, A. Figà-Talamanca and M. Yor, Non-symmetric hitting distributions on the hyperbolic half-plane and subordinated perpetuities, Rev. Mat. Iberoamer. 17 (2001), 587-605.

[B] P. Bougerol, Exemples de théorèmes locaux sur les groupes résolubles, Ann. Inst. H. Poincaré 19 (1983), 369-391.

[BGS] T. Byczkowski, P. Graczyk and A. Stós, Poisson kernels of half-spaces in real hyperbolic spaces, Rev. Mat. Iberoamer. (to appear); http//arxiv.org/math.PR/ 0503372 .

[D] D. Dufresne, The distribution of a perpetuity, with applications to risk theory and pension funding, Scand. Actuar. J. 1990, 39-79.

[E] A. Erdélyi et al. (eds.), Higher Transcendental Functions, Vol. II, McGraw-Hill, New York, 1953-1955.

[Fe] W. Feller, An Introduction to Probability Theory and Its Applications, Vol. II, 2nd ed., Wiley, New York, 1971.

[G] R. K. Getoor, The Brownian escape process, Ann. Probab. 7 (1979), 864-867.

[GS] R. K. Getoor and M. J. Sharpe, Excursions of Brownian motion and Bessel processes, Z. Wahrsch. Verw. Gebiete 47 (1979), 83-106.

[GJY] A. Göing-Jaeschke and M. Yor, A survey and some generalizations of Bessel processes, Bernoulli 9 (2003), 313-349. 
[GY] H. Geman and M. Yor, Bessel processes, Asian options and perpetuities, Math. Finance 3 (1993), 349-375.

[I1] M. E. Ismail, Bessel functions and the infinite divisibility of the Student $t$ distribution, Ann. Probab. 5 (1977), 582-585.

[I2] - Integral representations and complete monotonicity of various quotients of Bessel functions, Canad. J. Math. 29 (1977), 1198-1207.

[IK] M. E. Ismail and D. H. Kelker, Special functions, Stieltjes transforms and infinite divisibility, SIAM J. Math. Anal. 10 (1976), 884-901.

[K1] J. Kent, Some probabilistic properties of Bessel functions, Ann. Probab. 6 (1978), 760-770.

[K2] -, Eigenvalue expansions for diffusion hitting times, Z. Wahrsch. Verw. Gebiete 52 (1980), 309-319.

[K3] -, The spectral decomposition of a diffusion hitting time, Ann. Probab. 10 (1982), 207-219.

[L] J. Lamperti, Semi-stable Markov processes I, Z. Wahrsch. Verw. Gebiete 22 (1972), 205-225.

[M] C. Monthus, Étude de quelques fonctionnelles du movement Brownien et de certaines propriétés de la diffusion unidimensionnelle en milieu aléatoire, Thése de Doctorat de l'Univ. Paris VI, 1995.

[PY] J. Pitman and M. Yor, Bessel processes and infinitely divisible laws, in: Stochastic Integrals, D. Williams (ed.), Lecture Notes in Math. 851, Springer, 1981, 285-370.

[PY1] - - - Processus de Bessel, et mouvement brownien, avec "drift", C. R. Acad. Sci. Paris Sér. A 291 (1980), 151-153.

[RY] D. Revuz and M. Yor, Continuous Martingales and Brownian Motion, 2nd ed., Springer, 1994.

[Sa] K. Sato, Lévy Processes and Infinitely Divisible Distributions, Cambridge Univ. Press, Cambridge, 1999.

[S] F. Spitzer, Some theorems concerning 2-dimensional Brownian motion, Trans. Amer. Math. Soc. 87 (1958), 187-197.

[Y1] M. Yor, Sur certaines fonctionnelles du mouvement Brownien réel, J. Appl. Probab. 29 (1992), 202-208.

[Y2] - On some exponential functionals of Brownian motion, Adv. Appl. Probab. 24 (1992), 509-531.

[Y3] M. Yor (ed.), Exponential Functionals and Principal Values Related to Brownian Motion, Bibl. Rev. Mat. Iberoamer., Madrid, 1997.

Institute of Mathematics and Informatics

Wrocław University of Technology

Wybrzeże Wyspiańskiego 27

50-370 Wrocław, Poland

E-mail: tomasz.byczkowski@pwr.wroc.pl

Received February 10, 2005

Revised version July 12, 2005 